\title{
A model of relative translation and rotation in leader-follower spacecraft formations
}

\author{
Raymond Kristiansen ${ }^{1}$ Esten I. Grøtli ${ }^{2}$ Per J. Nicklasson ${ }^{1}$ Jan T. Gravdahl ${ }^{2}$ \\ ${ }^{1}$ Department of Computer Science, Electrical Engineering and Space Technology, Narvik University College, N-8505 Narvik, \\ Norway. E-mail: $\{$ rayk,pjn\}@hin.no \\ ${ }^{2}$ Department of Engineering Cybernetics, Norwegian University of Science and Technology, N-7491 Trondheim, Norway. E- \\ mail: $\{$ Esten.Grotli,Tommy.Gravdahl\}@itk.ntnu.no
}

\begin{abstract}
In this paper, a model of a leader-follower spacecraft formation in six degrees of freedom is derived and presented. The nonlinear model describes the relative translational and rotational motion of the spacecraft, and extends previous work by providing a more complete factorization, together with detailed information about the matrices in the model. The resulting model shows many similarities with models for systems such as robot manipulators and marine vehicles. In addition, mathematical models of orbital perturbations due to gravitational variations, atmospheric drag, solar radiation and third-body effects are presented for completeness. Results from simulations are presented to visualize the properties of the model and to show the impact of the different orbital perturbations on the flight path.
\end{abstract}

Keywords: Spacecraft formation, relative motion, 3D general orbits, orbital perturbations

\section{Introduction}

\subsection{Background}

The concept of flying spacecraft in formation is revolutionizing our way of performing space-based operations, and this new paradigm brings out several advantages in space mission accomplishment and extends the possible application area for such systems. Spacecraft formation flying is a technology that includes two or more spacecraft in a controlled spatial configuration, whose operations are closely synchronized, and Earth and deep space surveillance are areas where spacecraft formations can be useful. These applications often involve data collection and processing over an aperture where the resolution of the observation is inversely proportional to the baseline lengths. Further exploration of neighboring galaxies in space can only be achieved by indirect observation of astronomical objects, and space based interferometers with baselines of up to ten kilometers have been proposed. However, to successfully utilize space- craft formations for this purpose, accurate synchronization of both position and attitude of the cooperating spacecraft is vital, depending on accurate dynamical system models of the formation.

\subsection{Previous work}

The simplest model of relative motion between two spacecraft is linear and multi-variable, and known as the Hill or Clohessy-Wiltshire equations (Hill, 1878; Clohessy and Wiltshire, 1960). This model originated from the equations of the two-body problem, based on the laws of Newton and Kepler, and has inherently assumptions that the orbit is circular with no orbital perturbations, and that the distance between spacecraft is small relative to the distance from the formation to the center of the Earth. An extension to elliptic Keplerian orbits, yet still assuming no orbital perturbations, is what is known as the Lawden equations (Lawden, 1954) or also Tschauner-Hempel equations (Tschauner, 1967). Both models were originally presented 
for solutions of the problem of orbital rendezvous, but have been adopted later for the related and more general spacecraft formation flying control problem. As the visions for tighter spacecraft formations in highly elliptic orbits appeared, the need for more detailed models arose, especially regarding orbital perturbations. This resulted in nonlinear models as presented in e.g. McInnes (1995); Wang and Hadaegh (1996), and later in Manikonda et al. (1999) and Yan et al. (2000), derived for arbitrary orbital eccentricity and with added terms for orbital perturbations.

Alternative approaches for modelling spacecraft formations are the method of orbit element differences (Schaub et al., 1999, 2000; Schaub, 2004) and Theona theory (Golikov, 2003). The first originates from Lagrange and Gauss equations, and is based on the idea that each spacecraft in the formation will have a desired orbit described by a specific set of orbit parameters. The orbital perturbations will then cause the orbital parameters for each spacecraft to drift away from the desired parameters, and this is known as orbit element differences. The strength of this method in a control perspective is that the spacecraft are controlled relative to their natural orbits, instead of keeping the formation fixed as in the Newtonian approach. However, control of orbit element differences requires orbit determination and global positioning, which can often be computationally demanding, and the accuracy needed for close formation flying is hard to achieve. In Newtonian models, control is only dependent of relative positions and velocities in the formation, which can be acquired with high accuracy by means of optical or radar-based inter-satellite links (ILS).

The numeric-analytic Theona satellite theory is a computationally efficient orbit propagation method used with success for optimal maneuver and station keeping of spacecraft formations. Similar to orbit element differences, this approach is based on orbital parameters, but Theona theory is a mathematical extension that can include more corrections in satellite motion.

Models of both translational and rotational motion in a leader-follower spacecraft formation have been considered by few researchers, and most of the previous work has focused on translational models only. However, notable exceptions are Wang and Hadaegh (1996); Pan and Kapila (2001), where coupled models of translation and rotation were derived. In Naasz et al. (2003), a 6DOF model based on orbit element differences was derived in order to develop an integrated control system for attitude and orbit control. A coordinate-free model of translation and rotation for a single spacecraft in a formation was presented in several different forms in Ploen et al. (2004).

\subsection{Contribution}

This paper is an extension of Kristiansen et al. (2005), and presents a detailed nonlinear mathematical model in six de- grees of freedom of relative translational and rotational motion of two spacecraft in a leader-follower formation, which is well suited for control. The model of relative position is based on the two-body equations derived from Newton's inverse square law of gravity, and extends previous work by providing a more complete factorization, together with detailed information about the matrices in the model. The position and velocity vectors of the follower spacecraft are represented in a coordinate reference frame located in the center of mass of the leader spacecraft, known as the Hill frame. The relative attitude model is based on Euler's momentum equations, and the attitude is represented by unit quaternions and angular velocities. As the title of the paper indicates, the presented model is tailored for relative translational and rotational motion for leader-follower spacecraft formation. It should however be noted that the model can also be used to describe the relative translational motion of general orbits in space.

The model also includes the mathematical expressions for orbital perturbations originating from gravitational variations, atmospheric drag, solar radiation, and perturbations due to other celestial bodies, known as third body effects.

The rest of the paper is organized as follows: Section 2 describes the reference coordinate frames used in the paper, and matrices for vector rotation between frames. In Section 3 the model of relative position and velocity is derived, and the model of relative attitude and angular velocity is derived in Section 4. The models for relative translation and rotation are summarized into a total nonlinear model in Section 5. Expressions for orbital perturbations are given in Section 6. Simulation results for a spacecraft formation are presented in Section 7, and concluding remarks can be found in Section 8.

\section{Coordinate frames}

\subsection{Cartesian coordinate frames}

The coordinate reference frames used throughout the paper are given in Figure 1 and defined as follows:

Earth Centered Inertial (ECI) frame: This frame is denoted $\mathcal{F}_{i}$, and has its origin located in the center of the Earth. Its $z$ axis is directed along the rotation axis of the Earth towards the celestial north pole, the $x$ axis is directed towards the vernal equinox, and finally the direction of the $y$ axis completes a right handed orthogonal frame.

Leader orbit reference frame: The leader orbit frame, denoted $\mathcal{F}_{l}$, has its origin located in the center of mass of the leader spacecraft. The $\mathbf{e}_{r}$ axis in the frame is parallel to the vector $\mathbf{r}_{l}$ pointing from the center of the Earth to the spacecraft, and the $\mathbf{e}_{h}$ axis is parallel to the orbit momentum vector, which points in the orbit normal direction. The $\mathbf{e}_{\theta}$ axis completes the right-handed orthogonal frame. The 


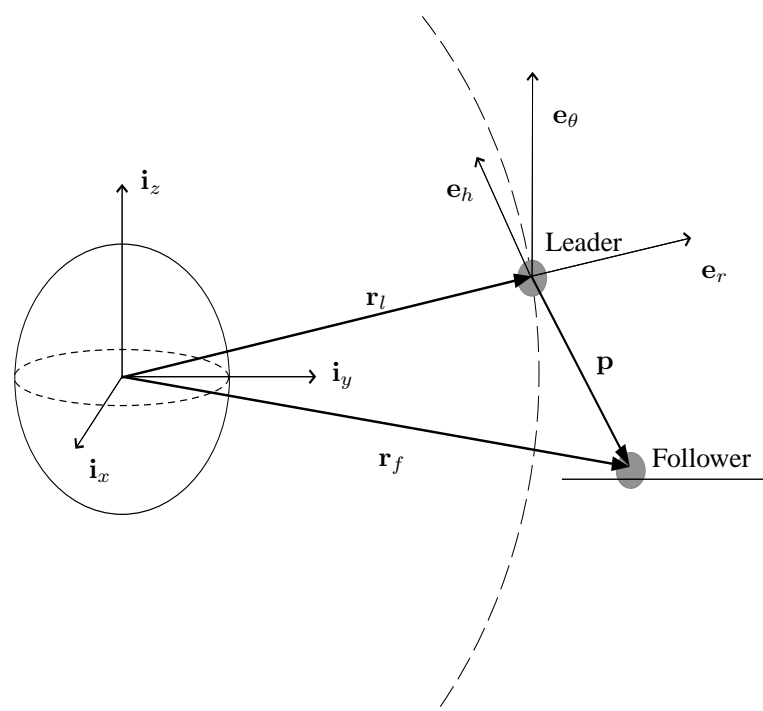

Figure 1: Reference coordinate frames (Schaub and Junkins, 2003).

basis vectors of the frame can be defined as

$$
\mathbf{e}_{r}=\frac{\mathbf{r}_{l}}{r_{l}}, \quad \mathbf{e}_{\theta}=\mathbf{e}_{h} \times \mathbf{e}_{r} \quad \text { and } \quad \mathbf{e}_{h}=\frac{\mathbf{h}}{h},
$$

where $\mathbf{h}=\mathbf{r}_{l} \times \dot{\mathbf{r}}_{l}$ is the angular momentum vector of the orbit, and $h=|\mathbf{h}|$.

In addition to the basis vectors of the frame $\mathcal{F}_{l}$, two auxiliary vectors $\mathbf{e}_{v}$ and $\mathbf{e}_{n}$ are defined, as shown in Figure 2. The first vector $\mathbf{e}_{v}$ is pointing along the spacecraft velocity vector, while $\mathbf{e}_{n}$ is defined to be orthogonal to $\mathbf{e}_{v}$ and $\mathbf{e}_{h}$, as $\mathbf{e}_{n}=\mathbf{e}_{v} \times \mathbf{e}_{h}$. If the spacecraft orbit is circular, then $\mathbf{e}_{v}=\mathbf{e}_{\theta}$ and $\mathbf{e}_{n}=\mathbf{e}_{r}$. The auxiliary vector frame is used when incorporating models for atmospheric drag, which has a resultant force in the $-\mathbf{e}_{v}$ direction.

Follower orbit reference frame: This frame has its origin in the center of mass of the follower spacecraft, and is denoted $\mathcal{F}_{f}$. The vector pointing from the center of the Earth to the center of the follower orbit frame is denoted $\mathbf{r}_{f}$. Its origin is specified by a relative orbit position vector $\mathbf{p}=\left[\begin{array}{lll}x & y & z\end{array}\right]^{T}$ expressed in $\mathcal{F}_{l}$ frame components, as shown in Figure 1, and the frame unit vectors align with the basis vectors of $\mathcal{F}_{l}$. Accordingly,

$$
\mathbf{p}=\mathbf{r}_{f}-\mathbf{r}_{l}=x \mathbf{e}_{r}+y \mathbf{e}_{\theta}+z \mathbf{e}_{h}
$$

Body reference frames: For both the leader and the follower spacecraft, body reference frames are defined and denoted $\mathcal{F}_{b l}$ and $\mathcal{F}_{b f}$, respectively. These frames have, similar to the orbit frame, the origin located in the center of mass of the respective spacecraft, but the basis vectors are fixed in the spacecraft body and coincide with its principal axis of inertia.

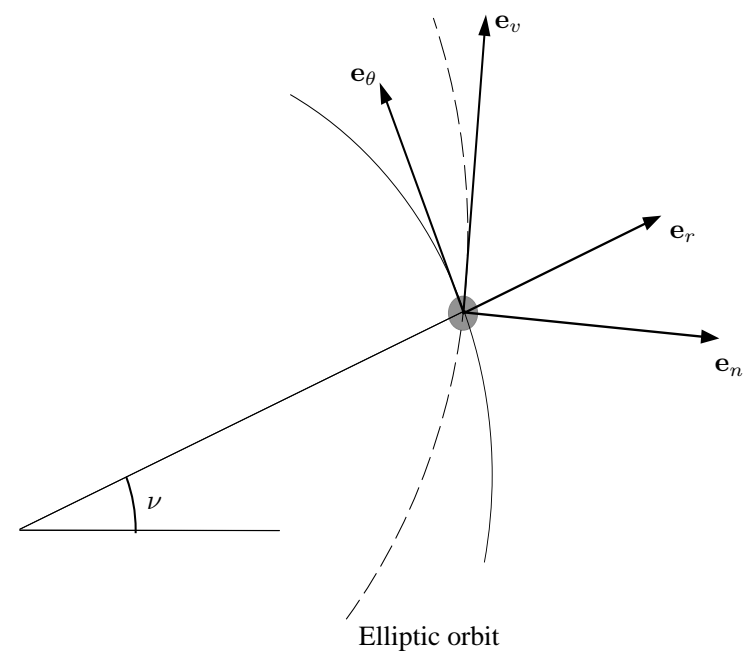

Figure 2: Auxiliary vectors for the leader orbit reference frame (Schaub and Junkins, 2003).

\subsection{Coordinate frame transformations}

\subsubsection{Rotation from $\mathrm{ECl}$ to leader orbit frame}

The rotation from the ECI frame to the leader orbit frame is dependent on the parameters of the leader spacecraft orbit, and can be expressed by three consecutive rotations. The total rotation matrix can be written

$$
\mathbf{R}_{i}^{l}=\mathbf{R}_{z, \omega+\nu} \mathbf{R}_{x, i} \mathbf{R}_{z, \Omega}
$$

where $\Omega$ is the right ascension of the ascending node of the orbit, $i$ is the inclination of the orbit, $\nu$ is the true anomaly of the leader orbit, and $\omega$ is the argument of perigee of the same. The sum of $\nu$ and $\omega$ represents the location of the spacecraft relative to the ascending node.

\subsubsection{Orbit frame transformation}

Using both the original and the auxiliary vectors in the orbit frame, as shown in Figure 2, spacecraft acceleration can be written as

$$
\mathbf{a}=a_{r} \mathbf{e}_{r}+a_{\theta} \mathbf{e}_{\theta}+a_{h} \mathbf{e}_{h}=a_{n} \mathbf{e}_{n}+a_{v} \mathbf{e}_{v}+a_{h} \mathbf{e}_{h} .
$$

The spacecraft velocity vector can be expressed according to Schaub and Junkins (2003) as

$$
\mathbf{v}_{s}=\dot{\mathbf{r}}=\frac{\mu}{h}\left(e \sin \nu \mathbf{e}_{r}+\frac{p}{r} \mathbf{e}_{\theta}\right)
$$

where $\mu$ is the geocentric gravitational constant of the Earth, $h$ is the magnitude of angular momentum, $e$ is the eccentricity and $p=h^{2} / \mu$ is the semi-latus rectum of the spacecraft 
orbit. Therefore, since $\mathbf{e}_{v}$ is pointing along the velocity vector,

$$
\mathbf{e}_{v}=\frac{\mathbf{v}_{s}}{\left|\mathbf{v}_{s}\right|}=\frac{h}{p v}\left(e \sin \nu \mathbf{e}_{r}+\frac{p}{r} \mathbf{e}_{\theta}\right) .
$$

Moreover, since $\mathbf{e}_{n}$ is defined normal to $\mathbf{e}_{v}$ and $\mathbf{e}_{h}$,

$$
\mathbf{e}_{n}=\mathbf{e}_{v} \times \mathbf{e}_{h}=\frac{h}{p v}\left(\frac{p}{r} \mathbf{e}_{r}-e \sin \nu \mathbf{e}_{\theta}\right) .
$$

The coordinate transformation between the orbit plane acceleration vector components can now be found from (2)(4) as

$$
\left[\begin{array}{l}
a_{r} \\
a_{\theta}
\end{array}\right]=\frac{h}{p v}\left[\begin{array}{cc}
\frac{p}{r} & e \sin \nu \\
-e \sin \nu & \frac{p}{r}
\end{array}\right]\left[\begin{array}{l}
a_{n} \\
a_{v}
\end{array}\right]
$$

so that

$$
\mathbf{C}_{a}^{l}=\frac{h}{p v}\left[\begin{array}{ccc}
\frac{p}{r} & e \sin \nu & 0 \\
-e \sin \nu & \frac{p}{r} & 0 \\
0 & 0 & \frac{p v}{h}
\end{array}\right]
$$

Note that $\mathbf{C}_{a}^{l}$ is not in general a proper rotation matrix since

$$
\operatorname{det} \mathbf{C}_{a}^{l}=1+e^{2}+2 e \cos \nu
$$

\subsubsection{Body frame rotation}

The rotation matrix describing rotations from an orbit frame to a body frame can be described by

$$
\mathbf{R}_{o}^{b}=\left[\mathbf{c}_{1} \mathbf{c}_{2} \mathbf{c}_{3}\right]=\mathbf{I}+2 \eta \mathbf{S}(\boldsymbol{\epsilon})+2 \mathbf{S}^{2}(\boldsymbol{\epsilon})
$$

where the elements $\mathbf{c}_{i}$ are directional cosines, and

$$
\mathbf{q}=\left[\begin{array}{ll}
\eta & \boldsymbol{\epsilon}^{T}
\end{array}\right]^{T}
$$

are the Euler parameters. The matrix $\mathbf{S}(\cdot)$ is the cross product operator given by

$$
\mathbf{S}(\boldsymbol{\epsilon})=\boldsymbol{\epsilon} \times=\left[\begin{array}{ccc}
0 & -\epsilon_{z} & \epsilon_{y} \\
\epsilon_{z} & 0 & -\epsilon_{x} \\
-\epsilon_{y} & \epsilon_{x} & 0
\end{array}\right]
$$

when $\boldsymbol{\epsilon}=\left[\epsilon_{x} \epsilon_{y} \epsilon_{z}\right]^{T}$. The inverse rotation is given by the complex conjugate of $\mathbf{q}$ as

$$
\overline{\mathbf{q}}=\left[\begin{array}{ll}
\eta & -\boldsymbol{\epsilon}^{T}
\end{array}\right]^{T} .
$$

\section{Relative translation}

\subsection{The $\mathrm{N}$-body problem}

Consider a system of $N$ bodies with masses $m_{i}$, where $i=$ $1,2, \ldots, N$. The position and velocity vectors of the $i$ 'th mass relative to the ECI frame $\mathcal{F}_{i}$ are defined as $\mathbf{r}_{i}$ and $\mathbf{v}_{i}$ respectively, where $\mathbf{r}_{i}=x_{i} \mathbf{i}_{x}+y_{i} \mathbf{i}_{y}+z_{i} \mathbf{i}_{z}$ and $\mathbf{v}_{i}=\frac{d \mathbf{r}_{i}}{d t}$. The distance between any two particles with mass $m_{i}$ and $m_{j}$ is denoted by

$$
r_{i j}=\left|\mathbf{r}_{j}-\mathbf{r}_{i}\right|
$$

and the magnitude of the force of attraction between the masses is $G m_{i} m_{j} / r_{i j}^{2}$ where $G$ is the universal constant of gravity (Battin, 1999). The direction of the forces are expressed in terms of unit vectors, and the force acting on $m_{i}$ due to $m_{j}$ has the direction $\left(\mathbf{r}_{j}-\mathbf{r}_{i}\right) / r_{i j}$, while the force on $m_{j}$ due to $m_{i}$ has the opposite direction. The force $\mathbf{f}_{i}$ acting on mass $m_{i}$ due to all the other $N-1$ masses can be expressed as

$$
\mathbf{f}_{i}=G \sum_{j=1}^{n} \frac{m_{i} m_{j}}{r_{i j}^{3}}\left(\mathbf{r}_{j}-\mathbf{r}_{i}\right), \quad i, j=1,2, \ldots, N, \quad i \neq j
$$

and application of Newton's second law of motion yields $N$ vector differential equations

$$
\frac{d^{2} \mathbf{r}_{i}}{d t^{2}}=G \sum_{j=1}^{n} \frac{m_{j}}{r_{i j}^{3}}\left(\mathbf{r}_{j}-\mathbf{r}_{i}\right), \quad i \neq j
$$

Together with appropriate initial conditions, this constitutes a complete mathematical description of the motion of a system of $N$ bodies. From this relation, the fundamental differential equation of the two-body problem can be found as (cf. Battin (1999))

$$
\frac{d^{2} \mathbf{r}}{d t^{2}}+\frac{\mu}{r^{3}} \mathbf{r}=\mathbf{0}
$$

where $\mathbf{r}=\mathbf{r}_{2}-\mathbf{r}_{1}$ is the relative position of masses and $\mu=G\left(m_{1}+m_{2}\right)$.

\subsection{Formation dynamics}

The general orbit equation (10) is the equation describing the orbit dynamics for a spacecraft under ideal conditions, i.e. with no orbital perturbations. This equation can be generalized to include force terms due to aerodynamic disturbances, gravitational forces from other bodies, solar radiation, magnetic fields and so on. In addition, it can be augmented to include control input vectors from onboard actuators. Accordingly, (10) can be expressed for the leader and follower spacecraft as

$$
\begin{gathered}
\ddot{\mathbf{r}}_{l}=-\frac{\mu}{r_{l}^{3}} \mathbf{r}_{l}+\frac{\mathbf{f}_{d l}}{m_{l}}+\frac{\mathbf{f}_{a l}}{m_{l}} \\
\ddot{\mathbf{r}}_{f}=-\frac{\mu}{r_{f}^{3}} \mathbf{r}_{f}+\frac{\mathbf{f}_{d f}}{m_{f}}+\frac{\mathbf{f}_{a f}}{m_{f}}
\end{gathered}
$$

where $\mathbf{f}_{d l}, \mathbf{f}_{d f} \in \mathbb{R}^{3}$ are the orbital perturbation terms due to external effects and $\mathbf{f}_{a l}, \mathbf{f}_{a f} \in \mathbb{R}^{3}$ are the actuator forces of the leader and follower spacecraft, respectively. In 
addition, spacecraft masses are assumed to be small relative to the mass of the Earth $M_{e}$, so $\mu=G M_{e}$. The second order derivative of the relative position vector can now be expressed as

$$
\begin{aligned}
\ddot{\mathbf{p}} & =\ddot{\mathbf{r}}_{f}-\ddot{\mathbf{r}}_{l} \\
& =-\frac{\mu}{r_{f}^{3}} \mathbf{r}_{f}+\frac{\mathbf{f}_{d f}}{m_{f}}+\frac{\mathbf{f}_{a f}}{m_{f}}+\frac{\mu}{r_{l}^{3}} \mathbf{r}_{l}-\frac{\mathbf{f}_{d l}}{m_{l}}-\frac{\mathbf{f}_{a l}}{m_{l}}
\end{aligned}
$$

so that

$$
\begin{aligned}
m_{f} \ddot{\mathbf{p}}=-m_{f} \mu\left(\frac{\mathbf{r}_{l}+\mathbf{p}}{\left(r_{l}+p\right)^{3}}-\frac{\mathbf{r}_{l}}{r_{l}^{3}}\right) & \\
& \quad+\mathbf{f}_{a f}+\mathbf{f}_{d f}-\frac{m_{f}}{m_{l}}\left(\mathbf{f}_{a l}+\mathbf{f}_{d l}\right) .
\end{aligned}
$$

On the other hand, from (1), the dynamics of the follower spacecraft relative to the leader spacecraft, referenced in the leader orbit frame $\mathcal{F}_{l}$, can be expressed as

$$
\mathbf{r}_{f}=\mathbf{r}_{l}+\mathbf{p}=\left(r_{l}+x\right) \mathbf{e}_{r}+y \mathbf{e}_{\theta}+z \mathbf{e}_{h} .
$$

Differentiation of this equation twice with respect to time leaves

$$
\begin{aligned}
\ddot{\mathbf{r}}_{f}=\left(\ddot{r}_{l}+\ddot{x}\right) \mathbf{e}_{r}+2\left(\dot{r}_{l}+\dot{x}\right) \dot{\mathbf{e}}_{r}+\left(r_{l}+x\right) \ddot{\mathbf{e}}_{r} \\
+\ddot{y} \mathbf{e}_{\theta}+2 \dot{y} \dot{\mathbf{e}}_{\theta}+y \ddot{\mathbf{e}}_{\theta}+\ddot{z} \mathbf{e}_{h}+2 \dot{z} \dot{\mathbf{e}}_{h}+z \ddot{\mathbf{e}}_{h} .
\end{aligned}
$$

By using the true anomaly $\nu$ of the leader spacecraft, the relationships

$$
\begin{array}{ll}
\dot{\mathbf{e}}_{r}=\dot{\nu} \mathbf{e}_{\theta} & \dot{\mathbf{e}}_{\theta}=-\dot{\nu} \mathbf{e}_{r} \\
\ddot{\mathbf{e}}_{r}=\ddot{\nu} \mathbf{e}_{\theta}-\dot{\nu}^{2} \mathbf{e}_{r} & \ddot{\mathbf{e}}_{\theta}=-\ddot{\nu} \mathbf{e}_{r}-\dot{\nu}^{2} \mathbf{e}_{\theta}
\end{array}
$$

can be found. Insertion of (13)-(14) into (12), while recognizing that no out-of-plane motion exists in the ideal case, and hence $\dot{\mathbf{e}}_{h}=\ddot{\mathbf{e}}_{h}=\mathbf{0}$, gives

$$
\begin{aligned}
\ddot{\mathbf{r}}_{f}= & \left(\ddot{r}_{l}+\ddot{x}-2 \dot{y} \dot{\nu}-\dot{\nu}^{2}\left(r_{l}+x\right)-y \ddot{\nu}\right) \mathbf{e}_{r} \\
& +\left(\ddot{y}+2 \dot{\nu}\left(\dot{r}_{l}+\dot{x}\right)+\ddot{\nu}\left(r_{l}+x\right)-y \dot{\nu}^{2}\right) \mathbf{e}_{\theta}+\ddot{z} \mathbf{e}_{h} .
\end{aligned}
$$

Moreover, the position of the leader spacecraft can be expressed as $\mathbf{r}_{l}=r_{l} \mathbf{e}_{r}$, and differentiating this expression twice with respect to time and inserting (13)-(14), results in

$$
\begin{aligned}
\ddot{\mathbf{r}}_{l} & =\ddot{r}_{l} \mathbf{e}_{r}+2 \dot{r}_{l} \dot{\mathbf{e}}_{r}+r_{l} \ddot{\mathbf{e}}_{r} \\
& =\left(\ddot{r}_{l}-r_{l} \dot{\nu}^{2}\right) \mathbf{e}_{r}+\left(2 \dot{r}_{l} \dot{\nu}+r_{l} \ddot{\nu}\right) \mathbf{e}_{\theta} .
\end{aligned}
$$

Subtracting (16) from (15) results thus in the formulation of the position vector acceleration represented in the $\mathcal{F}_{l}$ frame;

$$
\begin{aligned}
\ddot{\mathbf{p}}= & \ddot{\mathbf{r}}_{f}-\ddot{\mathbf{r}}_{l} \\
=(\ddot{x}- & \left.2 \dot{\nu} \dot{y}-\dot{\nu}^{2} x-\ddot{\nu} y\right) \mathbf{e}_{r} \\
& \quad+\left(\ddot{y}+2 \dot{\nu} \dot{x}+\ddot{\nu} x-\dot{\nu}^{2} y\right) \mathbf{e}_{\theta}+\ddot{z} \mathbf{e}_{h} .
\end{aligned}
$$

Substituting (17) into (11), and denoting relative velocity as $\mathbf{v}=\dot{\mathbf{p}}$, leaves the nonlinear position dynamics on the form

$$
\begin{aligned}
m_{f} \dot{\mathbf{v}}+\mathbf{C}_{t}(\dot{\nu}) \mathbf{v}+\mathbf{D}_{t} & \left(\dot{\nu}, \ddot{\nu}, r_{f}\right) \mathbf{p} \\
& +\mathbf{n}_{t}\left(r_{l}, r_{f}\right)=\mathbf{F}_{a}+\mathbf{F}_{d}
\end{aligned}
$$

similar to the one derived in Yan et al. (2000), where

$$
\mathbf{C}_{t}(\dot{\nu})=2 m_{f} \dot{\nu}\left[\begin{array}{ccc}
0 & -1 & 0 \\
1 & 0 & 0 \\
0 & 0 & 0
\end{array}\right] \in S S(3)
$$

is a skew-symmetric Coriolis-like matrix,

$$
\mathbf{D}_{t}\left(\dot{\nu}, \ddot{\nu}, r_{f}\right) \mathbf{p}=m_{f}\left[\begin{array}{cccc}
\frac{\mu}{r_{f}^{3}}-\dot{\nu}^{2} & -\ddot{\nu} & 0 \\
\ddot{\nu} & \frac{\mu}{r_{f}^{3}}-\dot{\nu}^{2} & 0 \\
0 & 0 & \frac{\mu}{r_{f}^{3}}
\end{array}\right] \mathbf{p}
$$

may be viewed as a time-varying potential force, and

$$
\mathbf{n}_{t}\left(r_{l}, r_{f}\right)=m_{f} \mu\left[\begin{array}{c}
\frac{r_{l}}{r_{f}^{3}}-\frac{1}{r_{l}^{2}} \\
0 \\
0
\end{array}\right] \text {. }
$$

The composite perturbation force $\mathbf{F}_{d}$ is given by

$$
\mathbf{F}_{d}=\mathbf{f}_{d f}-\frac{m_{f}}{m_{l}} \mathbf{f}_{d l}
$$

and the relative control force $\mathbf{F}_{a}$ is given by

$$
\mathbf{F}_{a}=\mathbf{f}_{a f}-\frac{m_{f}}{m_{l}} \mathbf{f}_{a l}
$$

The eigenvalues of the matrix $\mathbf{D}_{t}\left(\dot{\nu}, \ddot{\nu}, r_{f}\right)$ in (19) are

$$
\lambda=\left\{\frac{\mu}{r_{f}^{3}}+\ddot{\nu}-\dot{\nu}^{2} \quad \frac{\mu}{r_{f}^{3}}-\ddot{\nu}-\dot{\nu}^{2} \quad \frac{\mu}{r_{f}^{3}}\right\}
$$

so it can be shown that $\mathbf{D}_{t}\left(\dot{\nu}, \ddot{\nu}, r_{f}\right)>0$ when

$$
r_{f}^{3}<\frac{a^{3}\left(1-e^{2}\right)^{3}}{(1+e \cos \nu)^{4}}
$$

where $a$ is the semimajor axis of the leader orbit. If the orbit of the leader spacecraft is circular, then $e=0$ and (20) reduces to $r_{f}<a$, so $\mathbf{D}_{t}\left(\dot{\nu}, \ddot{\nu}, r_{f}\right)>0$ when the follower is located within the circle with origin in the center of the Earth and radius $a$. At the other end, when the leader orbit tends towards an parabolic orbit, then $e \rightarrow 1$ and (20) reduces to $r_{f}<0$, which is practically infeasible. For control purposes, it is desirable to have $\mathbf{D}_{t}\left(\dot{\nu}, \ddot{\nu}, r_{f}\right) \leq 0$, as this would constitute a "tether-like" connection between the leader and the follower. However, forcing the follower spacecraft to satisfy such a constraint will lead to unnatural orbital behavior for the follower and hence increase the consumption of power. 


\section{Relative rotation}

\subsection{Attitude kinematics}

The time derivative of a matrix $\mathbf{R}_{b}^{a}$ as in (6) can according to Egeland and Gravdahl (2002) be written as

$$
\dot{\mathbf{R}}_{b}^{a}=\mathbf{S}\left(\boldsymbol{\omega}_{a, b}^{a}\right) \mathbf{R}_{b}^{a}=\mathbf{R}_{b}^{a} \mathbf{S}\left(\boldsymbol{\omega}_{a, b}^{b}\right)
$$

where $\boldsymbol{\omega}_{a, b}^{b}$ is the angular velocity of frame $b$ relative to frame $a$ represented in frame $b$ and $\mathbf{S}(\cdot)$ is the cross product operator described in (8). The kinematic differential equations for a spacecraft in its orbit frame can be found from (21) together with (7) as

$$
\dot{\mathbf{q}}_{s}=\left[\begin{array}{c}
\dot{\eta}_{s} \\
\dot{\boldsymbol{\epsilon}}_{s}
\end{array}\right]=\frac{1}{2}\left[\begin{array}{c}
-\boldsymbol{\epsilon}_{s}^{T} \\
\eta_{s} \mathbf{I}+\mathbf{S}\left(\boldsymbol{\epsilon}_{s}\right)
\end{array}\right] \boldsymbol{\omega}_{s, s b}^{s b}
$$

where $\boldsymbol{\omega}_{s, s b}^{s b}$ is the angular velocity of the spacecraft body frame relative to the orbit frame, referenced in the body frame. The superscript/subscript $s$ is used in general to denote the spacecraft in question, so $s=l, f$ for the leader and follower spacecraft, respectively.

\subsection{Attitude dynamics}

With the assumptions of rigid body movement, the dynamical model of a spacecraft can be found from Euler's momentum equation as ( $c f$. Sidi (1997))

$$
\begin{aligned}
\mathbf{J}_{s} \dot{\boldsymbol{\omega}}_{i, s b}^{s b} & =-\mathbf{S}\left(\boldsymbol{\omega}_{i, s b}^{s b}\right) \mathbf{J}_{s} \boldsymbol{\omega}_{i, s b}^{s b}+\boldsymbol{\tau}_{d s}^{s b}+\boldsymbol{\tau}_{a s}^{s b} \\
\boldsymbol{\omega}_{s, s b}^{s b} & =\boldsymbol{\omega}_{i, s b}^{s b}+\omega_{o} \mathbf{c}_{2}
\end{aligned}
$$

where $\mathbf{J}_{s}$ is the spacecraft inertia matrix and $\boldsymbol{\omega}_{i, s b}^{s b}$ is the angular velocity of the spacecraft body frame relative to the inertial frame, expressed in the body frame. The parameter $\omega_{o}$ is the orbit angular velocity, $\boldsymbol{\tau}_{d}^{s b}$ is the orbital perturbation torque, $\boldsymbol{\tau}_{a}^{s b}$ is the actuator torque, and $\mathbf{c}_{2}$ is the directional cosine vector from (6).

\subsection{Relative attitude}

By expressing the relations in (22) and (23)-(24) for both the leader and the follower spacecraft, and using the quaternion product defined in Egeland and Gravdahl (2002) as

$$
\mathbf{q}=\mathbf{q}_{f} \otimes \overline{\mathbf{q}}_{l} \triangleq\left[\begin{array}{c}
\eta_{f} \eta_{l}+\boldsymbol{\epsilon}_{f}^{T} \boldsymbol{\epsilon}_{l} \\
\eta_{l} \boldsymbol{\epsilon}_{f}-\eta_{f} \boldsymbol{\epsilon}_{l}-\mathbf{S}\left(\boldsymbol{\epsilon}_{f}\right) \boldsymbol{\epsilon}_{l}
\end{array}\right]
$$

the relative attitude kinematics and dynamics can be expressed as ( $c f$. Fjellstad (1994))

$$
\dot{\mathbf{q}}=\left[\begin{array}{c}
\dot{\eta} \\
\dot{\boldsymbol{\epsilon}}
\end{array}\right]=\frac{1}{2}\left[\begin{array}{c}
-\boldsymbol{\epsilon}^{T} \\
\eta \mathbf{I}+\mathbf{S}(\boldsymbol{\epsilon})
\end{array}\right] \boldsymbol{\omega}
$$

where

$$
\boldsymbol{\omega}=\boldsymbol{\omega}_{i, f b}^{f b}-\mathbf{R}_{l b}^{f b} \boldsymbol{\omega}_{i, l b}^{l b}
$$

is the relative angular velocity between the leader body reference frame and the follower body reference frame. Moreover, from (25) the relative attitude dynamics can be expressed as

$$
\begin{aligned}
\mathbf{J}_{f} \dot{\boldsymbol{\omega}} & =\mathbf{J}_{f} \dot{\boldsymbol{\omega}}_{i, f b}^{f b}-\mathbf{J}_{f} \dot{\mathbf{R}}_{l b}^{f b} \boldsymbol{\omega}_{i, l b}^{l b}-\mathbf{J}_{f} \mathbf{R}_{l b}^{f b} \dot{\boldsymbol{\omega}}_{i, l b}^{l b} \\
& =\mathbf{J}_{f} \dot{\boldsymbol{\omega}}_{i, f b}^{f b}-\mathbf{J}_{f} \mathbf{S}\left(\boldsymbol{\omega}_{i, l b}^{f b}\right) \boldsymbol{\omega}-\mathbf{J}_{f} \mathbf{R}_{l b}^{f b} \dot{\boldsymbol{\omega}}_{i, l b}^{l b}
\end{aligned}
$$

where (21) and the facts that $\boldsymbol{\omega}_{l b, f b}^{f b}=\boldsymbol{\omega}$ and $\mathbf{S}(\mathbf{a}) \mathbf{b}=$ $-\mathbf{S}(\mathbf{b})$ a have been used. Insertion of (23), evaluated for both the leader and follower, into (26) results in

$$
\begin{aligned}
& \mathbf{J}_{f} \dot{\boldsymbol{\omega}}+\mathbf{J}_{f} \mathbf{S}\left(\mathbf{R}_{l b}^{f b} \boldsymbol{\omega}_{i, l b}^{l b}\right) \boldsymbol{\omega}-\mathbf{J}_{f} \mathbf{R}_{l b}^{f b} \mathbf{J}_{l}^{-1} \mathbf{S}\left(\boldsymbol{\omega}_{i, l b}^{l b}\right) \mathbf{J}_{l} \boldsymbol{\omega}_{i, l b}^{l b} \\
& +\mathbf{S}\left(\boldsymbol{\omega}+\mathbf{R}_{l b}^{f b} \boldsymbol{\omega}_{i, l b}^{l b}\right) \mathbf{J}_{f}\left(\boldsymbol{\omega}+\mathbf{R}_{l b}^{f b} \boldsymbol{\omega}_{i, l b}^{l b}\right)=\boldsymbol{\Upsilon}_{d}+\boldsymbol{\Upsilon}_{a}
\end{aligned}
$$

where

$$
\boldsymbol{\Upsilon}_{d}=\boldsymbol{\tau}_{d f}^{f b}-\mathbf{J}_{f} \mathbf{R}_{l b}^{f b} \mathbf{J}_{l}^{-1} \boldsymbol{\tau}_{d l}^{l b}
$$

and

$$
\boldsymbol{\Upsilon}_{a}=\boldsymbol{\tau}_{a f}^{f b}-\mathbf{J}_{f} \mathbf{R}_{l b}^{f b} \mathbf{J}_{l}^{-1} \boldsymbol{\tau}_{a l}^{l b}
$$

are the relative orbital perturbation torques and relative actuator torques, respectively. The third term in (27), denoted $\boldsymbol{\xi}(\boldsymbol{\omega})$, can be rewritten as

$$
\begin{gathered}
\mathbf{S}\left(\boldsymbol{\omega}+\mathbf{R}_{l b}^{f b} \boldsymbol{\omega}_{i, l b}^{l b}\right) \mathbf{J}_{f}\left(\boldsymbol{\omega}+\mathbf{R}_{l b}^{f b} \boldsymbol{\omega}_{i, l b}^{l b}\right) \\
=\mathbf{S}(\boldsymbol{\omega}) \mathbf{J}_{f}\left(\boldsymbol{\omega}+\mathbf{R}_{l b}^{f b} \boldsymbol{\omega}_{i, l b}^{l b}\right) \\
\quad+\mathbf{S}\left(\mathbf{R}_{l b}^{f b} \boldsymbol{\omega}_{i, l b}^{l b}\right) \mathbf{J}_{f}\left(\boldsymbol{\omega}+\mathbf{R}_{l b}^{f b} \boldsymbol{\omega}_{i, l b}^{l b}\right) \\
=-\mathbf{S}\left(\mathbf{J}_{f}\left(\boldsymbol{\omega}+\mathbf{R}_{l b}^{f b} \boldsymbol{\omega}_{i, l b}^{l b}\right)\right) \boldsymbol{\omega} \\
\quad+\mathbf{S}\left(\mathbf{R}_{l b}^{f b} \boldsymbol{\omega}_{i, l b}^{l b}\right) \mathbf{J}_{f}\left(\boldsymbol{\omega}+\mathbf{R}_{l b}^{f b} \boldsymbol{\omega}_{i, l b}^{l b}\right) \\
=\left(\begin{array}{r}
\left.-\mathbf{S}\left(\mathbf{J}_{f}\left(\boldsymbol{\omega}+\mathbf{R}_{l b}^{f b} \boldsymbol{\omega}_{i, l b}^{l b}\right)\right)+\mathbf{S}\left(\mathbf{R}_{l b}^{f b} \boldsymbol{\omega}_{i, l b}^{l b}\right) \mathbf{J}_{f}\right) \boldsymbol{\omega} \\
\quad+\mathbf{S}\left(\mathbf{R}_{l b}^{f b} \boldsymbol{\omega}_{i, l b}^{l b}\right) \mathbf{J}_{f} \mathbf{R}_{l b}^{f b} \boldsymbol{\omega}_{i, l b}^{l b}
\end{array}\right.
\end{gathered}
$$

and accordingly, (27) can be written as

$$
\mathbf{J}_{f} \dot{\boldsymbol{\omega}}+\mathbf{C}_{r}(\boldsymbol{\omega}) \boldsymbol{\omega}+\mathbf{n}_{r}(\boldsymbol{\omega})=\mathbf{\Upsilon}_{d}+\mathbf{\Upsilon}_{a}
$$

where

$$
\begin{aligned}
\mathbf{C}_{r}(\boldsymbol{\omega})=\mathbf{J}_{f} \mathbf{S}\left(\mathbf{R}_{l b}^{f b} \boldsymbol{\omega}_{i, l b}^{l b}\right)+ & \mathbf{S}\left(\mathbf{R}_{l b}^{f b} \boldsymbol{\omega}_{i, l b}^{l b}\right) \mathbf{J}_{f} \\
& -\mathbf{S}\left(\mathbf{J}_{f}\left(\boldsymbol{\omega}+\mathbf{R}_{l b}^{f b} \boldsymbol{\omega}_{i, l b}^{l b}\right)\right)
\end{aligned}
$$

is a skew-symmetric matrix, $\mathbf{C}_{r}(\boldsymbol{\omega}) \in S S(3)$, and

$$
\begin{aligned}
\mathbf{n}_{r}(\boldsymbol{\omega})=\mathbf{S}\left(\mathbf{R}_{l b}^{f b} \boldsymbol{\omega}_{i, l b}^{l b}\right) & \mathbf{J}_{f} \mathbf{R}_{l b}^{f b} \boldsymbol{\omega}_{i, l b}^{l b} \\
& -\mathbf{J}_{f} \mathbf{R}_{l b}^{f b} \mathbf{J}_{l}^{-1} \mathbf{S}\left(\boldsymbol{\omega}_{i, l b}^{l b}\right) \mathbf{J}_{l} \boldsymbol{\omega}_{i, l b}^{l b}
\end{aligned}
$$


is a nonlinear term. The skew-symmetry of $\mathbf{C}_{r}(\boldsymbol{\omega})$ can be shown as follows: The sum of the first and last term in (29) is skew-symmetric, since $\mathbf{J}_{f}=\mathbf{J}_{f}^{T}$ and $\mathbf{S}(\cdot)=-\mathbf{S}^{T}(\cdot)$, so

$$
\mathbf{J}_{f} \mathbf{S}(\cdot)+\mathbf{S}(\cdot) \mathbf{J}_{f}+\left[\mathbf{J}_{f} \mathbf{S}(\cdot)+\mathbf{S}(\cdot) \mathbf{J}_{f}\right]^{T}=0 .
$$

Moreover, the second term in (29) is itself skew-symmetric, and the sum of two skew-symmetric matrices is also skewsymmetric. Accordingly, $\mathbf{C}_{r}(\boldsymbol{\omega})$ will be skew-symmetric.

\section{Total model}

To write the total 6DOF model of relative translation and rotation in the spacecraft formation, define the state vectors

$$
\mathbf{x}_{1}=[\mathbf{p} \mathbf{q}]^{T} \quad \text { and } \quad \mathbf{x}_{2}=[\mathbf{v} \boldsymbol{\omega}]^{T} .
$$

Based on (18) and (28), the total model of the relative translational and rotational motion between the leader and the follower spacecraft can now be expressed

$$
\begin{aligned}
\dot{\mathbf{x}}_{1} & =\boldsymbol{\Lambda}\left(\mathbf{x}_{1}\right) \mathbf{x}_{2} \\
\mathbf{M}_{f} \dot{\mathbf{x}}_{2} & =\mathbf{U}+\mathbf{W}-\mathbf{C}(\dot{\nu}, \boldsymbol{\omega}) \mathbf{x}_{2} \\
& \quad-\mathbf{D}\left(\dot{\nu}, \ddot{\nu}, r_{f}\right) \mathbf{x}_{1}-\mathbf{n}\left(\boldsymbol{\omega}, r_{l}, r_{f}\right)
\end{aligned}
$$

where

$$
\mathbf{M}_{f}=\left[\begin{array}{cc}
m_{f} \mathbf{I} & \mathbf{0} \\
\mathbf{0} & \mathbf{J}_{f}
\end{array}\right]
$$

is a symmetric positive definite matrix containing the mass and moments of inertia of the follower spacecraft,

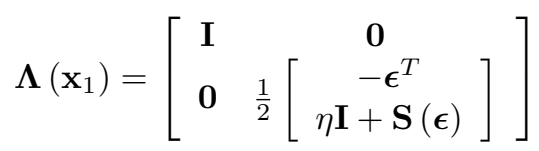

is the coupling term between the first and second order dynamics,

$$
\mathbf{C}(\dot{\nu}, \boldsymbol{\omega})=\left[\begin{array}{cc}
\mathbf{C}_{t}(\dot{\nu}) & \mathbf{0} \\
\mathbf{0} & \mathbf{C}_{r}(\boldsymbol{\omega})
\end{array}\right] \in S S(6)
$$

is the skew-symmetric Coriolis-like matrix,

$$
\mathbf{D}\left(\dot{\nu}, \ddot{\nu}, r_{f}\right) \mathbf{x}_{1}=\left[\begin{array}{cc}
\mathbf{D}_{t}\left(\dot{\nu}, \ddot{\nu}, r_{f}\right) & \mathbf{0} \\
\mathbf{0} & \mathbf{0}
\end{array}\right] \mathbf{x}_{1}
$$

is the time-varying potential force term,

$$
\mathbf{n}\left(\boldsymbol{\omega}, r_{l}, r_{f}\right)=\left[\begin{array}{c}
\mathbf{n}_{t}\left(r_{l}, r_{f}\right) \\
\mathbf{n}_{r}(\boldsymbol{\omega})
\end{array}\right]
$$

is the composite nonlinear term, and finally

$$
\mathbf{U}=\left[\begin{array}{c}
\mathbf{F}_{a} \\
\mathbf{\Upsilon}_{a}
\end{array}\right] \quad \text { and } \quad \mathbf{W}=\left[\begin{array}{c}
\mathbf{F}_{d} \\
\mathbf{\Upsilon}_{d}
\end{array}\right]
$$

contains the relative input forces and orbital perturbations, respectively. It should be noted that when orbital perturbations and actuator torque is neglected, the relative rotational and translational motion of the leader spacecraft and the follower spacecraft is uncoupled. Equation (31) can be interpreted as a "mass-damper-spring" system with an added nonlinear term, and the model has many similarities with models of systems such as robot manipulators, and surface and underwater ships ( $c f$. Ortega et al. (1998); Fossen (2002)). This is convenient since automatic control theory for these types of systems is highly developed, and this simplifies the task of controller design.

\section{Orbital perturbations}

Spacecraft flying in a Keplerian orbit will be subject to accelerations caused by orbital perturbation forces. The main sources for these forces are gravitational attractions from celestial bodies, non-spherical planets, atmospheric drag, or solar radiation pressure (Schaub and Junkins, 2003). The resulting expressions for these perturbations are in the following presented generally for a spacecraft in Earth orbit, without relating to leader or follower spacecraft. The superscript $s$ is used for generality to indicate the orbit frame for the inflicted spacecraft.

\subsection{Perturbing forces}

\subsubsection{Atmospheric drag}

At altitudes lower than approximately $500 \mathrm{~km}$, Earth atmosphere is so dense that the resulting aerodynamic drag has a considerable impact on spacecraft orbits (Wertz, 1978). The aerodynamic force can be written as

$$
\mathbf{f}_{a t m}^{s}=\mathbf{C}_{a}^{s}\left[\begin{array}{c}
0 \\
\frac{1}{2} \rho V^{2} C_{d} A \\
0
\end{array}\right]
$$

where $\rho$ is the atmospheric density, $V$ is the spacecraft velocity, $C_{d}$ is the drag coefficient, $A$ is the equivalent spacecraft surface in the direction of motion of the spacecraft and $\mathbf{C}_{a}^{s}$ denotes the orbit frame transformation matrix, as described in (5).

\subsubsection{Solar radiation}

Radiation and particles expelled from the Sun will affect the spacecraft orbit independent of the spacecraft altitude (Wertz, 1978). The orbital perturbation force from solar radiation is dependent on the reflectance of the spacecraft material, and consists of absorption, specular reflection and diffuse reflection. The surfaces of a spacecraft is usually non-diffuse, and the reflection is a combination of absorption and specular reflection. The diffuse reflection is hence 
neglected in the further analysis. A visualization of resulting forces on a surface $A$ due to absorption and specular reflection is shown in Figure 3. The normal vector $\mathbf{n}$ in the

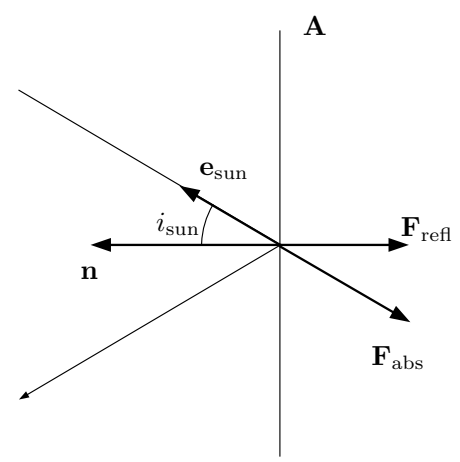

Figure 3: Orbital perturbation forces due to solar radiation pressure for absorbing and reflecting surfaces.

figure gives the orientation of the surface $A$, and it is inclined an angle $i_{\text {sun }}$ to the vector $\mathbf{e}_{\text {sun }}$ which points in the direction of the Sun. For an absorbing surface, the impulse transferred is in the opposite direction as $\mathbf{e}_{\text {sun }}$. For a reflecting surface on the other hand, the impulse transferred is not generally in the opposite direction as $\mathbf{e}_{\text {sun }}$, and the impulse is also twice as large, due to the reflective rays. For a body that reflects a fraction $\gamma$ of the incoming radiation, while it absorbs the remaining fraction of energy $(1-\gamma)$, the total combined force is given as

$$
\mathbf{f}_{\text {sun }}^{s}=\frac{F_{\text {sun }}}{c} \cos i_{\text {sun }} A\left[(\gamma-1) \mathbf{e}_{\text {sun }}-2 \gamma \cos i_{\text {sun }} \mathbf{n}\right]
$$

where $F_{\text {sun }}=1367 \mathrm{~W} / \mathrm{m}^{2}$ is the solar constant and $c$ is the speed of light.

\subsubsection{The mass distribution of the Earth}

If the Earth was a single point mass, the gravitational potential due to the conservative gravitational force could be derived from a gradient or scalar potential function $U(r)=$ $-\mu / r$. However, the Earth is not a single point mass, but an oblate body with a nonhomogeneous mass distribution. Therefore, correction factors must be added based on the geographical position of the spacecraft, and the corrected potential of the Earth can be expressed according to Wertz (1978) as

$$
U(r, \phi, \lambda)=-\mu / r+B(r, \phi, \lambda)
$$

where $B(r, \phi, \lambda)$ is a spherical harmonic expansion, $\phi$ is the geocentric latitude and $\lambda$ is the geographical longitude of the spacecraft position. Denoting $R_{e}$ as the mean equatorial radius of the Earth, the expansion can be expressed as

$$
B(r, \phi, \lambda)=\frac{\mu}{r}\left\{\sum_{n=2}^{\infty}\left[H_{1, n}+\sum_{m=1}^{n} H_{2, n}\right]\right\}
$$

where

$$
\begin{aligned}
H_{1, n} & =\left(\frac{R_{e}}{r}\right)^{n} J_{n} P_{n}(\sin \phi) \\
H_{2, n} & =\left(\frac{R_{e}}{r}\right)^{n}\left(C_{n m} \cos m \lambda+S_{n m} \sin m \lambda\right) P_{n m}(\sin \phi)
\end{aligned}
$$

which is the infinite series of the geopotential function at any point $P$ outside of the Earth sphere where $r, \phi$ and $\lambda$ are its spherical coordinates (Sidi, 1997). The parameters $J_{n}$ are zonal harmonic coefficients, $P_{n m}$ are Legendre polynomials of degree $n$ and order $m, P_{n}=P_{n 0}$, and $C_{n m}$ and $S_{n m}$ are tesseral harmonic coefficients for $n \neq m$ and sectoral harmonic coefficients for $n=m$ (Wertz, 1978). Specifically, it should be noted that $J_{n} \equiv C_{n 0}$. The associated Legendre polynomial $P_{n m}$ is defined as ( $c f$. Montenbruck and Gill (2001))

$$
P_{n m}(u)=\left(1-u^{2}\right)^{\frac{m}{2}} \frac{d^{m}}{d u^{m}} P_{n}(u)
$$

It is seen from equation (34) that zonal harmonics depend only on latitude, not on longitude, and these coefficients are due to the oblateness of the Earth. The tesseral harmonics in (34) represents longitudinal variations in the Earth shape, and are generally smaller than zonal terms. A set of values for the $J_{n}$ constants are given in Table 1 . If the assumption

Table 1: Zonal harmonic coefficients (Roy, 2005)

\begin{tabular}{cc}
\hline$n$ & $J_{n}$ \\
\hline 2 & $1082.6 \cdot 10^{-6}$ \\
3 & $-2.51 \cdot 10^{-6}$ \\
4 & $-1.60 \cdot 10^{-6}$ \\
\hline
\end{tabular}

of axial symmetry of the Earth is introduced, only zonal harmonics is needed. In addition, from Table 1 of zonal harmonics coefficients, it is seen that $J_{2}$ is considerably larger than the other $J_{n}$ coefficients. If $J_{2}$ is the only zonal harmonic considered, the gravitational potential function can be approximated according to Montenbruck and Gill (2001) as

$$
U(r, \phi, \lambda)=\frac{\mu}{r}\left[-1+\frac{1}{2} J_{2}\left(\frac{R_{e}}{r}\right)^{2}\left(3 \sin ^{2} \phi-1\right)\right] .
$$

In the inertial reference frame,

$$
\sin \phi=\frac{r_{z}}{|\mathbf{r}|}=\frac{r_{z}}{\sqrt{r_{x}^{2}+r_{y}^{2}+r_{z}^{2}}}
$$

where $\mathbf{r}$ is the vector pointing from the center of the Earth to the spacecraft. The gravitational force $\mathbf{G}$ acting on the spacecraft is obtained from the gradient of the scalar poten- 
tial as

$$
\mathbf{G}=\mu\left[\begin{array}{c}
-\frac{r_{x}}{r^{3}}+\frac{1}{2} J_{2} R_{e}^{2}\left(15 \frac{r_{x} r_{z}^{2}}{r^{7}}-3 \frac{r_{x}}{r^{5}}\right) \\
-\frac{r_{y}}{r^{3}}+\frac{1}{2} J_{2} R_{e}^{2}\left(15 \frac{r_{y} r_{z}^{2}}{r^{7}}-3 \frac{r_{y}}{r^{5}}\right) \\
-\frac{r_{z}}{r^{3}}+\frac{1}{2} J_{2} R_{e}^{2}\left(15 \frac{r_{z}^{3}}{r^{7}}-9 \frac{r_{z}}{r^{5}}\right)
\end{array}\right]
$$

and the $J_{2}$ gravity perturbation force $\mathbf{f}_{\text {grav }}^{b}$ for the Earth is the latter terms in (35), i.e.

$$
\mathbf{f}_{\text {grav }}^{s}=\frac{3}{2} \mu J_{2} R_{e}^{2} \mathbf{R}_{i}^{s}\left[\begin{array}{c}
5 \frac{r_{x} r_{z}^{2}}{r^{7}}-\frac{r_{x}}{r^{5}} \\
5 \frac{r_{y} r_{z}^{2}}{r^{7}}-\frac{r_{y}}{r^{5}} \\
5 \frac{r_{z}^{3}}{r^{7}}-3 \frac{r_{z}}{r^{5}}
\end{array}\right] .
$$

\subsubsection{Third-body perturbing forces}

The gravitational potential of other bodies in the vicinity of the spacecraft can create perturbing forces and torques. For an Earth-orbiting spacecraft, the Sun and the Moon causes perturbing forces that can change the orbit parameters considerably. The Keplerian orbit models are derived from the two-body problem equation in (10), after a simplification of the general equation (9) due to the assumption the spacecraft and the orbited planet are the only elements present. If an extraction of the masses of the spacecraft and the Earth is performed on (9), the resulting accelerations are ( $c f$. Battin (1999))

$$
\begin{aligned}
\frac{d^{2} \mathbf{r}_{1}}{d t^{2}} & =G \frac{m_{2}}{r_{12}^{3}}\left(\mathbf{r}_{2}-\mathbf{r}_{1}\right)+G \sum_{j=3}^{n} \frac{m_{j}}{r_{1 j}^{3}}\left(\mathbf{r}_{j}-\mathbf{r}_{1}\right) \\
\frac{d^{2} \mathbf{r}_{2}}{d t^{2}} & =G \frac{m_{1}}{r_{21}^{3}}\left(\mathbf{r}_{1}-\mathbf{r}_{2}\right)+G \sum_{j=3}^{n} \frac{m_{j}}{r_{2 j}^{3}}\left(\mathbf{r}_{j}-\mathbf{r}_{2}\right) .
\end{aligned}
$$

Subtraction of (37) from (38) results in the equation for the two-body problem in (10) with an additional perturbing acceleration due to the $n-2$ perturbing bodies,

$$
\frac{d^{2} \mathbf{r}}{d t^{2}}+\frac{\mu}{r^{3}} \mathbf{r}=G \sum_{j=3}^{n} m_{j}\left(\frac{\mathbf{r}_{2 j}}{r_{2 j}^{3}}-\frac{\mathbf{r}_{1 j}}{r_{1 j}^{3}}\right)
$$

where, as before, $\mathbf{r}=\mathbf{r}_{2}-\mathbf{r}_{1}$ is the relative position of the two primary masses, and $\mathbf{r}_{1 j}=\mathbf{r}_{j}-\mathbf{r}_{1}$ and $\mathbf{r}_{2 j}=\mathbf{r}_{j}-\mathbf{r}_{2}$. Hence, the perturbing acceleration is

$$
\mathbf{f}_{n b o d y}^{s}=\mathbf{R}_{i}^{s} \sum_{j=3}^{n} \mu_{p j}\left(\frac{\mathbf{r}_{2 j}}{r_{2 j}^{3}}-\frac{\mathbf{r}_{1 j}}{r_{1 j}^{3}}\right)
$$

where $\mu_{p j}=G m_{j}$ is the gravity constant for the $j$ 'th perturbing body.

\subsection{Perturbing torques}

The resulting torque $\tau_{j}^{s}$ on a spacecraft due to a perturbing force $\mathbf{f}_{j}^{s}$ can be found from the relation ( $c f$. Egeland and
Gravdahl (2002))

$$
\boldsymbol{\tau}_{j}^{s}=\mathbf{r}_{c}^{s} \times \mathbf{f}_{j}^{s}
$$

where $\mathbf{r}_{c}^{s}$ is the vector from the spacecraft center of mass to the line of action of the force. Hence, perturbing torques due to atmospheric drag, solar radiation, gravity variations and third body effects can be found by combining (40) with (32), (33), (36) and (39), respectively.

\subsection{Dynamical effect of perturbations}

The dynamical effect of orbital perturbations on the spacecraft in the formation is a coupling of the rotational and translational motion of the follower spacecraft. Perturbing forces working on the follower are dependent on its position, and especially its altitude. The resulting torques due to these perturbing forces, which cause rotational motion of the spacecraft, will thus also depend on the position. Accordingly, the rotational motion is affected by the translational motion. Similar, the effect of perturbing forces due to atmospheric drag and solar radiation on the translational motion is dependent on the orientation of the spacecraft, varying with the size of the equivalent surface area in the force direction. Therefore, the translational motion of the follower spacecraft is affected by the rotational motion of the spacecraft. The result of orbital perturbations is thus a two way coupling in the model presented in (31). Also, use of non-conservative actuators as thrusters, magnetic rods and solar sails will in some actuator configurations cause both rotational and translational spacecraft motion.

\section{Simulations}

To illustrate the impact of the perturbing forces and torques, simulation results for two spacecraft in a leader-follower formation are presented. It should be noted that only the effects of the gravity force and atmospheric drag are included in the simulations. The reason for this is that solar radiation and the third-body effects are dependent on the location of the Sun and other celestial bodies. The effect of these perturbations will therefore vary, depending on the orbit parameters and time of the year.

For simplicity of simulation, both spacecraft have a total mass of $m=1 \mathrm{~kg}$ and their moments of inertia are $\mathbf{J}=\operatorname{diag}([0.06,0.06,0.003]) \mathrm{kgm}^{2}$. The leader spacecraft is assumed perfectly controlled in a circular orbit with inclination $22.5^{\circ}$ and altitude $250 \mathrm{~km}$, and with a constant attitude relative to the ECI frame. The follower spacecraft is located $10 \mathrm{~m}$ behind the leader in the along-track direction, with the same initial orbit velocity and attitude. The simulations were performed using a Runge-Kutta ODE solver.

The position and velocity of the follower relative to the leader are shown in Figure 4. Similarly, the relative attitude 

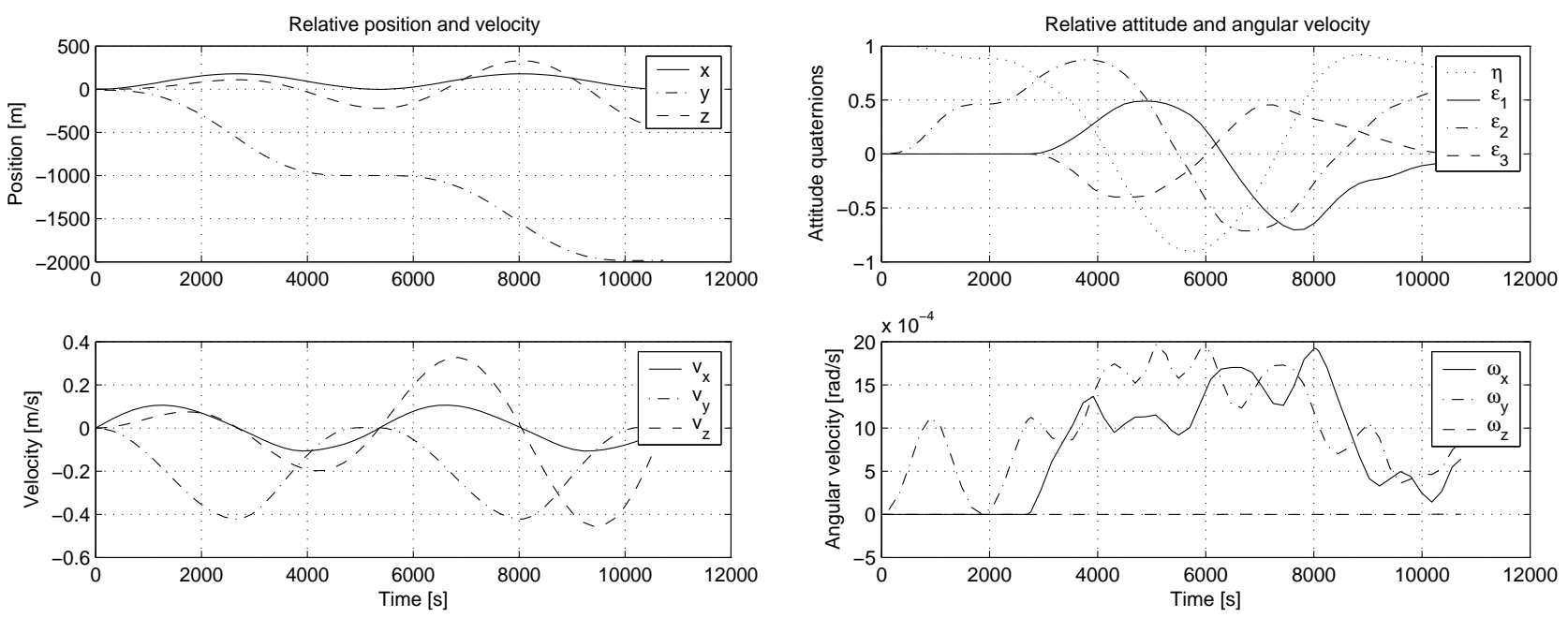

Figure 4: Relative position and velocity between leader and follower spacecraft.

Figure 5: Relative attitude and angular velocity between leader and follower spacecraft.

and angular velocity are presented in Figure 5. If no orbital perturbations were present, the relative position and attitude would be constant. Hence, the perturbing forces and torques can be seen from the figures to have a large impact on the system states. From the results presented in Figure 4, it can be seen that the perturbing forces causes oscillations in relative position. This is due to the gravity force working on the follower. The force pulls the spacecraft towards the Earth. However, as the follower moves below the leader, it has an orbital velocity corresponding to a higher orbit, and accordingly, the altitude increases. When it reaches the same altitude as the leader orbit, it is again drawn down towards the Earth, and the cycle repeats. Similar, the oscillations in the cross-track direction is due to gravity. Since the Earth is not a single point of mass, the follower will be drawn to the side with the largest gravitational pull. However, the main gravitational component will be towards the center of the Earth, so as the spacecraft moves to one side in cross-track direction, the gravitational force component in the opposite direction will pull it back again, and the increased cross-track velocity will move it over to the other side. As with the altitude, this is also a cyclic behavior, however, the cross-track motion seems to be more unstable. The along-track distance between the spacecraft was however not oscillating, but constantly decaying. The main cause of this is probably the atmospheric drag, which has considerable effect at altitudes below $500 \mathrm{~km}$. Hence, the along-track velocity of the follower is reduced. Also, oscillations in other directions cause the spacecraft to have a longer flight path, and thus it lags behind.

The relative attitude was also seen to oscillate. All three body axes were influenced by the perturbations, they had the largest effect on the $\mathbf{e}_{\theta}$ axis. This is due to the gravity force, which constantly tries to turn the follower towards

the Earth, in accordance with the principle axis of inertia. In addition, gravity perturbations originating from oblateness of the Earth results in non-smooth attitude changes, as shown in the simulation results.

Regarding the perturbations due to solar radiation and third-body effects, these are not included in the simulations. It is however possible to get a notion of the impact of these perturbations. Since the orbital time is short, the location of other celestial bodies like the Sun and the Moon can be considered constant over one orbit period. If these bodies are located in the orbital plane, they will result in a change in orbit eccentricity for the follower. The perturbing force due to solar radiation will decelerate the follower as it moves towards the Sun, and accelerate it as it moves away from the Sun. If the Sun is located out of the orbit plane, the follower will experience a constant force away from the Sun. The third-body effects have the opposite effect on the follower compared to the solar radiation, and accordingly, the spacecraft will experience a gravitational pull towards other celestial bodies.

\section{Conclusion}

In this paper, a nonlinear mathematical model of a leaderfollower spacecraft formation in six degrees of freedom has been derived and presented. The model describes the relative translational and rotational motions of the spacecraft, and extends previous work by providing a more complete factorization, together with detailed information about the matrices in the model. The resulting model shows many similarities with models for systems such as robot manipulators and marine vehicles. Moreover, mathematical models of orbital perturbations due to gravitational variations, at- 
mospheric drag, solar radiation and third-body effects have been included for completeness. Results from simulations of a leader-follower spacecraft formation have been presented to illustrate the effect of the orbital perturbations.

\section{References}

Battin, R. H. An Introduction to the Mathematics and Methods of Astrodynamics, Revised Edition. AIAA Education Series. American Institute of Aeronautics and Astronautics, Reston, VA, 1999.

Clohessy, W. H. and Wiltshire, R. S. Terminal guidance system for satellite rendezvous. Journal of Aerospace Sciences, 1960. 27(9):653-658.

Egeland, O. and Gravdahl, J. T. Modeling and Simulation for Automatic Control. Marine Cybernetics, Trondheim, Norway, 2002.

Fjellstad, O.-E. Control of Unmanned Underwater Vehicles in Six Degrees of Freedom. Ph.D. thesis, Department of Engineering Cybernetics, Norwegian University of Science and Technology, Trondheim, Norway, 1994.

Fossen, T. I. Marine Control Systems: Guidance, Navigation, and Control of Ships, Rigs and Underwater Vehicles. Marine Cybernetics, Trondheim, Norway, 2002.

Golikov, A. Evolution of formation flying satellite relative motion: analysis based on the theona satellite theory. In Proceedings of the 17th International Symposium on Space Flight Dynamics. Moscow, Russia, 2003.

Hill, G. W. Researches in the lunar theory. American Journal of Mathematics, 1878. 1(1):5-26.

Kristiansen, R., Grøtli, E. I., Nicklasson, P. J., and Gravdahl, J. T. A 6DOF model of a leader-follower spacecraft formation. In Proceedings of the Conference on Simulation and Modeling. Trondheim, Norway, 2005.

Lawden, D. Fundamentals of space navigation. Journal of the British Interplanetary Society, 1954. 13(2):87-101.

Manikonda, V., Arambel, P. O., Gopinathan, M., Mehra, R. K., and Hadaegh, F. Y. A model predictive controlbased approach for spacecraft formation keeping and attitude control. In Proceedings of the American Control Conference. San Diego, CA, 1999.

McInnes, C. R. Autonomous ring formation for a planar constellation of satellites. AIAA Journal of Guidance, Control and Dynamics, 1995. 18(5):1215-1217.

Montenbruck, O. and Gill, E. Satellite Orbits. Models, methods, applications. Springer-Verlag, Berlin, Germany, 2001. First edition, corrected second printing.
Naasz, B., Berry, M. M., Kim, H.-Y., and Hall, C. D. Integrated orbit and attitude control for a nanosatellite with power constraints. In Proceedings of the AAS/AIAA Space Flight Mechanics Conference. Ponce, Puerto Rico, 2003.

Ortega, R., Loría, A., Nicklasson, P. J., and Sira-Ramírez, H. Passivity-based Control of Euler-Lagrange Systems. Springer-Verlag, London, 1998.

Pan, H. and Kapila, V. Adaptive nonlinear control for spacecraft formation flying with coupled translational and attitude dynamics. In Proceedings of the Conference on Decision and Control. Orlando, FL, 2001 .

Ploen, S. R., Hadaegh, F. Y., and Scharf, D. P. Rigid body equations of motion for modeling and control of spacecraft formations - part 1: Absolute equations of motion. In Proceedings of the American Control Conference. Boston, MA, 2004 .

Roy, A. E. Orbital motion, Fourth edition. Institute of Physics Publishing Ltd, Bristol, UK, 2005.

Schaub, H. Relative orbit geometry through classical orbit element differences. AIAA Journal of Guidance, Control and Dynamics, 2004. 27(5):839-848.

Schaub, H. and Junkins, J. L. Analytical Mechanics of Space Systems. AIAA Education Series. American Institute of Aeronautics and Astronautics, Reston, VA, 2003.

Schaub, H., Vadali, S. R., Junkins, J. L., and Alfriend, K. T. Spacecraft formation control using mean orbit elements. Journal of the Astronautical Sciences, 1999. 48(1):6987.

Schaub, H., Vadali, S. R., Junkins, J. L., and Alfriend, K. T. Spacecraft formation flying control using mean orbit elements. Journal of the Astronautical Sciences, 2000. 48(1):69-87.

Sidi, M. J. Spacecraft Dynamics and Control. Cambridge University Press, New York, 1997.

Tschauner, J. Elliptic orbit rendezvous. AIAA Journal, 1967. 5(6):1110-1113.

Wang, P. K. C. and Hadaegh, F. Y. Coordination and control of multiple microspacecraft moving in formation. Journal of the Astronautical Sciences, 1996. 44(3):315-355.

Wertz, J. R., editor. Spacecraft Attitude Determination and Control. Kluwer Academic Publishers, London, 1978.

Yan, Q., Yang, G., Kapila, V., and de Queiroz, M. Nonlinear dynamics and adaptive control of multiple spacecraft in periodic relative orbits. In Proceedings of the AAS Guidance and Control Conference. Breckenridge, CO, 2000 